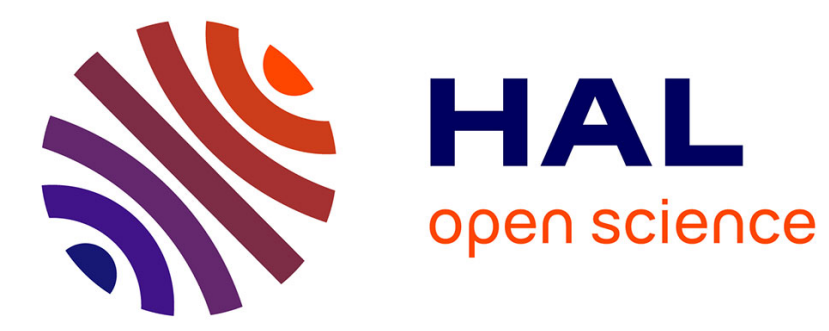

\title{
Thermally excited tunneling from a metastable electronic state in a single-Cooper-pair transistor
}

David G. Rees, Philip Glasson, Luke R. Simkins, Vladimir Antonov, Peter G. Frayne, Philip J. Meeson, Michael J. Lea, Eddy Collin

\section{- To cite this version:}

David G. Rees, Philip Glasson, Luke R. Simkins, Vladimir Antonov, Peter G. Frayne, et al.. Thermally excited tunneling from a metastable electronic state in a single-Cooper-pair transistor. Applied Physics Letters, 2008, 93, pp.173508. 10.1063/1.3012374 . hal-00917408

\section{HAL Id: hal-00917408 \\ https://hal.science/hal-00917408}

Submitted on 13 Dec 2013

HAL is a multi-disciplinary open access archive for the deposit and dissemination of scientific research documents, whether they are published or not. The documents may come from teaching and research institutions in France or abroad, or from public or private research centers.
L'archive ouverte pluridisciplinaire HAL, est destinée au dépôt et à la diffusion de documents scientifiques de niveau recherche, publiés ou non, émanant des établissements d'enseignement et de recherche français ou étrangers, des laboratoires publics ou privés. 


\title{
Thermally excited tunneling from a metastable electronic state in a single-Cooper-pair transistor
}

\author{
D.G.Rees ${ }^{\dagger}$, P.Glasson, L.R.Simkins, V.Antonov, P.G.Frayne, P.J.Meeson, M.J.Lea \\ (1) Department of Physics, Royal Holloway, University of London, Egham, Surrey, \\ TW20 OEX, UK.
}

\author{
and \\ E.Collin \\ (2) Institut Néel, CNRS \& Université J. Fourier, 25 rue des Martyrs, BP166, F-38042 \\ Grenoble Cedex 9, France.
}

31 August 2008; revised 7 October 2008.

Metastable electron traps and two-level systems are common in solid-state devices and lead to background charge movement and charge noise in single-electron and singleCooper-pair transistors. We present measurements of the real-time capture and escape of individual electrons in metastable trapped states at very low temperatures, leading to charge offsets close to $1 e$. The charge movement exhibits thermal excitation to a hysteretic tunneling transition. The temperature dependence and hysteresis can be explained by the coupling of a two-level system to a quasiparticle trap.

PACS numbers: 73.23.Hk, 72.20.Jv, 73.40.Rw

Corresponding author: M.J.Lea,m.lea@rhul.ac.uk

\footnotetext{
† Present address: Low Temperature Physics Laboratory, Riken, Hirosawa 2-1, Wako-shi, 351-0198 Japan.
} 
The detection and control of localised electrons in both intrinsic and fabricated traps in solid-state devices are major technical challenges. Single-electron transistors (SET) and single-Cooper-pair transistors (SCPT) are used as sensitive electrometers in charge qubits ${ }^{1,2}$, quantum dots $^{3}$ and Cooper-pair boxes ${ }^{4}$. But these devices exhibit intrinsic charge traps and two-level systems ${ }^{5,6,7,8}$, giving charge offsets, two-level fluctuators, hysteresis and $1 / f$ noise ${ }^{9}$, which limit their performance. Trapped electrons can escape from a metastable quantum state by tunneling ${ }^{10}$ through a potential barrier or thermal activation over the barrier at a temperature $T$. The escape rate is then proportional to $\exp (-E / k T)$ with activation energy $E$. In some systems, the potential barrier can be driven to zero ${ }^{11}$ by an external control parameter $v$ with $E \propto v^{\xi}$ and $\xi \geq 1$. Trapped electrons can also escape by phonon-assisted tunneling, or tunneling from thermally excited energy levels ${ }^{12}$. We now present measurements of the detailed dynamics of the capture and escape of individual electrons in metastable trapped states at very low temperatures in an SCPT. Individual electron transitions were detected and measured.

The devices, designed for applications in surface-state charge detection ${ }^{13}$, were fabricated with multiple metallic layers using e-beam lithography on a $\mathrm{Si} / \mathrm{SiO}_{2}$ substrate. Voltages applied through low pass filters and a $1 \mathrm{~m}$ length of thermocoax to a set of gate electrodes controlled the electrostatic potential of an $\mathrm{Al}_{-} \mathrm{AlO}_{x}-\mathrm{Al} \mathrm{SET}^{14}$, Figure 1(a). The source-drain current $I_{\mathrm{SD}}$, measured with a DL1211 current preamplifier, exhibited a Josephson-quasiparticle tunneling (JQP) peak and a dissipative Josephson branch, Figure 1(b). Coulomb blockade oscillations (CBO) were observed in $I_{\mathrm{SD}}$ when sweeping a gate voltage $V_{\mathrm{G}}$, due to capacitive coupling $C_{\mathrm{G}}$ to the SET island inducing a charge $Q=$ $-C_{\mathrm{G}} V_{\mathrm{G}}$. On the JQP peak, the $\mathrm{CBO}$ were periodic in $Q=1 e$, Figure 1(c). At small sourcedrain voltages $\left(V_{\mathrm{SD}}<25 \mu \mathrm{V}\right)$, large amplitude $\mathrm{CBO}(>400 \mathrm{pA})$ were $2 e$ periodic $^{15}$, Figure 1(c), when the superconducting island was in an even-parity state with no unpaired electrons ${ }^{16}$. The $2 e \mathrm{CBO}$ amplitude decreased above $250 \mathrm{mK}$, changing to $1 e$ periodicity, due to thermally excited quasiparticles ${ }^{16}$. The low noise $\mathrm{CBO}\left(5 \times 10^{-4} \mathrm{e} / \sqrt{\mathrm{Hz}}\right.$ at $100 \mathrm{~Hz}$ ) were stable for long periods. The gate electrodes G1 were strongly coupled with a $1 e \mathrm{CBO}$ period of $6.6 \mathrm{mV}$, while gate electrode $\mathrm{G} 2$ was weakly coupled with a period of $32.8 \mathrm{mV}$. 
The source-drain current $I_{\mathrm{SD}}$ exhibited sudden jumps, equivalent to CBO phase discontinuities, Figure 1(c), for both $1 e$ and $2 e$ oscillations induced by each of the gates, indicating sudden charge offsets. Over a large G1 sweep of $1500 \mathrm{mV}$, up to 14 jumps occurred, with a distribution of charge offsets $\delta Q$ from $0.80 e$ to $1.00 e$ and a mean of 0.90 e. Beyond each transition, the $\mathrm{CBO}$ were again stable until the gate voltage was reversed, and the charge offset was reset, though with some hysteresis. Small charge offsets, $|\delta Q|<0.2 e$, were sometimes observed but were not reproducible. Each reversible pair of transitions corresponds to electron capture and escape from a trap close to the SET island. In the low temperature limit, there is a threshold voltage $V_{+}$for electron capture (sweeping one gate voltage $V_{\mathrm{G}}$ positive, with other electrodes constant) and a different threshold $V_{-}$for electron escape (sweeping $V_{\mathrm{G}}$ negative). The control parameters for each transition are $v_{+}=V_{\mathrm{G}}-V_{+}$and $v_{-}=V_{-}-V_{\mathrm{G}}$, as $V_{\mathrm{G}}$ is swept. Hence $v<0$ corresponds to voltages before the threshold is reached and $v>0$ to voltages beyond the threshold. The hysteresis for each pair of transitions is $\Delta V_{ \pm}=V_{+}-V_{-}$. One specific transition was stable and reproducible, enabling detailed measurements of its dynamics.

We measured the real-time capture and escape rates, one electron at a time, by rapidly changing the gate voltage $V_{\mathrm{G} 2}$ from below the threshold $(v<<0$, stable initial state) to a constant value $V_{\mathrm{G} 2}$ above the threshold ( $v>0$, the now metastable initial state decays) and then measuring the time before the trap filled or emptied, as seen by a jump in $I_{\mathrm{SD}}$. The trap was reset by sweeping $V_{\mathrm{G} 2}$ back to the stable state below the threshold. The measurement was repeated to generate an ensemble of switching times $t_{i}(i=1$ to 1000). A plot of the remaining number of initial states versus $t_{i}$ gives a good exponential $\exp (-t / \tau)$ with a decay time $\tau$, Figure 2 . We plot $1 / \tau$ versus the gate voltage $V_{\mathrm{G} 2}$ at $25 \mathrm{mK}$, in both the electron capture and escape regions. Hysteresis occurs between $V_{-}$and $V_{+}$, with no charge movement $(1 / \tau=0)$. The data is asymmetric. A region of constant $1 / \tau$ occurs on both sides of the hysteresis region (dashed lines), but then $1 / \tau$ increases rapidly.

We also swept $V_{\mathrm{G} 2}$ through the threshold at a constant rate $\mathrm{d} V_{\mathrm{G} 2} / \mathrm{d} t= \pm a$ and measured the distribution of voltages at which 1000 individual transitions occurred, for a range of temperatures. Figure 3(a) shows the probability $P(v, T)$ of the occupancy of the initial state which decreases from 1 to 0 through the transition. The transition has a sharp 
threshold at low temperatures and broadens as $T$ increases. The rate of decrease of $P$ is $(1 / P) \mathrm{d} P / \mathrm{d} t=a \mathrm{~d} \ln P / \mathrm{d} v=-1 / \tau$. The slope of the $\log _{10} P$ vs. $v$ plot, Figure $3(\mathrm{~b})$, is proportional to $1 / \tau$, and is constant for $v>0$, above the threshold. Below the threshold ( $v$ $<0)$ an Arrhenius factor ${ }^{9}$ gives an excellent account of the escape and capture rates as the excitation energy $E=e|v| / \gamma$ increases linearly from zero, where $\gamma$ is a geometrical voltage scaling factor ${ }^{17}$. Experimentally, an excellent fit is found for

$$
\begin{array}{ll}
1 / \tau(v, T)=\left(1 / \tau_{0}\right) \exp (-E / k T) & \text { for } v<0 \\
1 / \tau(v, T)=1 / \tau_{0} & \text { for } v \geq 0
\end{array}
$$

where $1 / \tau_{0}$ is the value at and above the threshold, which can be integrated to give $P(v, T)$ :

$$
\begin{array}{ll}
P(v, T)=\exp \left[\left(-\gamma k T / e a \tau_{0}\right) \exp (e v / \gamma k T)\right] & \text { for } v<0 \\
P(v, T)=\exp \left(-\gamma k T / e a \tau_{0}\right) \exp \left(-v / a \tau_{0}\right) & \text { for } v \geq 0
\end{array}
$$

Fits to Eq.(2) are plotted in Figures 3(a) and (b) ${ }^{18}$. The nested exponentials in Eq.2(a) give a strong temperature dependence for $v<0$. A plot of $\log _{10}\left(-\log _{10} P\right)$ versus $v$ gives a straight line with a slope proportional to $1 / \gamma T$. The experimental temperature dependence of $\gamma T$ is shown in Figure 4. Above $50 \mathrm{mK}, \gamma T$ is proportional to $T$ with $\gamma=145 \pm 5$. Below $50 \mathrm{mK}, \gamma T$ lies above this line, as the electronic temperature of the SET reaches a minimum value $(\sim 35 \mathrm{mK})$ due to Joule heating ${ }^{19}$. The large value for $\gamma$ reflects the weak coupling of the gate to the small dipole moment of the charge excitation. The escape time $\tau_{0}(T)$ is almost independent of temperature, Figure 4 , and is long, $\approx 34 \mathrm{~ms}$, suggesting that the threshold is the onset of energetically allowed tunneling. Similar results are obtained from both parts of the hysteretic transition. By comparison, Buehler et al. ${ }^{20}$ measured the telegraph noise for a two-level fluctuator in an $r f$-SET with a switching time of $3.4 \mu \mathrm{s}$.

Consider first the magnitude of the charge offsets. A $1 e$ charge shift corresponds to a parity shift in the superconducting island. But the charge shifts were not precisely $1 e$. The jumps in Figure 1(c) are $0.92 \pm 0.02 e$. The sign of the induced charge is equivalent to an excess electron brought close to the island, inducing a reduced positive charge $\delta Q=$ $c_{1} e /\left(c_{1}+c_{2}\right)$ where $c_{1}$ and $c_{2}$ are the capacitive couplings of the electron to the SET island and elsewhere. If we used only the $1 e \mathrm{CBO}$, Figure $1(\mathrm{c})$, as in previous experiments ${ }^{6,7}$, we would wrongly interpret the jump size as $-0.08 e$. Random $1 e$ jumps were observed 
previously in an NSN device ${ }^{21}$, and were interpreted as the tunneling of electrons from the superconducting SET island to nearby traps. We conclude that there are quasiparticle traps close to the SET island, whose occupancy is limited by Coulomb blockade.

Furlan and Lotkhov ${ }^{6}$ investigated intrinsic charge noise in a normal state Al SET on oxidised Si and found bistable traps close to the SET island. Brown, Sun and Kane ${ }^{7}$ suggested that small isolated $\mathrm{Al}$ grains could act as quasiparticle traps. Fewer charge offsets are observed in a doped silicon $\mathrm{SET}^{22}$, with no metallic electrodes. Other traps or two-level systems may be associated with chemisorbed oxygen ions or oxygen clusters ${ }^{23}$ in the amorphous $\mathrm{AlO}_{x}$ layer.

The temperature dependent tunneling rate is revealing. Tunneling from a superconductor to small metallic particles was studied by Zeller and Giaever ${ }^{24}$ and through individual Al particles by Ralph, Black and Tinkham ${ }^{25}$. The tunneling rate $1 / \tau$ versus voltage is proportional to the superconducting density of states, and diverges at the energy gap. If the transitions in Figure 3 are tunneling to or from the superconducting island, then we should observe a maximum of $1 / \tau$ for $v \geq 0$, rather than the constant $1 / \tau_{0}$. However, Eq.(1) was used by Rogers and Buhrman ${ }^{9}$ and by Grupp et al. ${ }^{5}$ for the relaxation of an intrinsic two-level system (TLS).

We suggest the following to explain (i) charge offsets close to $1 e$, (ii) hysteresis and (iii) Arrhenius excitation. As any gate voltage $V_{\mathrm{G}}$ is swept, intrinsic TLS transitions will occur, with local charge movements ${ }^{6}$, but relatively small charge offsets, $|\delta Q|<0.1 e$. But each transition will change the Coulomb energy of other nearby traps and may trigger the tunneling of an electron between the SET island and a quasiparticle trap ${ }^{26}$, which may then stabilise the TLS, giving hysteresis. The excitation energies ${ }^{24}$ of the TLS, $E$, and the trap, $E_{1}$, will depend on $V_{\mathrm{G}}$, the TLS state $(M=0,1)$ and the trap occupancy $(N=0,1)$ as

$$
\begin{aligned}
& E=e\left(V_{+}-V_{\mathrm{G}}\right) / \gamma-N \Delta E \\
& E_{1}=e\left(V_{1}-V_{\mathrm{G}}\right) / \gamma_{1}-M \Delta E
\end{aligned}
$$

where $\gamma_{1}$ is a voltage scaling factor for the trap as $V_{\mathrm{G}}$ is swept and $\Delta E$ is the difference of the Coulomb interaction energy between an electron in the trap and an electron in the $M=$ 0 and the $M=1$ TLS states. As $V_{\mathrm{G}}$ is swept, the trap will fill, or the TLS switch, whenever $E$ or $E_{1}=0$, or thermal excitation occurs. Since both mechanisms are independently thermally excited, the sequence of events will depend on their relative 
energies and rates. If we start in the state $(M=0, N=0)$ and sweep $V_{\mathrm{G}}$ positive, the TLS will switch at $V_{+}$to state $(1,0)$. If this then makes $E_{1}<0$, the trap will rapidly fill $\left(<<\tau_{0}\right)$ to state $(1,1)$, giving $|\delta Q| \approx 1 e$, but also decreasing $E$ and stabilising the TLS. Sweeping $V_{\mathrm{G}}$ back negative, the TLS switches to state $(0,1)$ at $V_{-}$, triggering the trap emptying back to $(0,0)$. The voltage hysteresis $\Delta V_{ \pm}=\gamma \Delta E / e$. Thermal excitation will occur as a threshold is approached. A larger change in the gate voltage (Figure 2) may allow the trap to fill without triggering by the TLS. Other charge movements can also affect $\tau_{0}(T)$ and the threshold voltages. Further details will be given elsewhere.

In summary, the capture and escape of trapped electrons in an SET show charge offsets close to $1 e$, thermal excitation to a tunneling transition and hysteresis between metastable states. We suggest that both quasiparticle traps and two-level systems contribute to intrinsic charge movement in SCPTs, and that electrostatic coupling between traps can produce correlated charge movements and hysteresis. This model is consistent with, and could help to explain, previous experiments ${ }^{6,7}$.

We thank Denis Vion and Mark Dykman for discussions and F.Greenough, A.K.Betts and others for technical support. PJM gratefully acknowledges the hospitality and stimulation provided by Group Quantronique, CEA Saclay. The work was supported by the EPSRC and by Royal Holloway, University of London. 


\section{References}

${ }^{1}$ L.C.L.Hollenberg, A.S.Dzurak, C.Wellard, A.R.Hamilton, D.J.Reilly, G.J.Milburn and R.G.Clark, Phys.Rev. B 69, 113301 (2004).

${ }^{2}$ G. Papageorgiou, P.Glasson, K.Harabi, V.Antonov, E.Collin, P.Fozooni, P.G.Frayne, M.J.Lea, D.G.Rees and Y.Mukharsky, Appl.Phys.Lett. 86, 153106 (2005); E.Rousseau, Y.Mukharsky, D.Ponarine, O.Avenel and E.Varoquaux, J. Low Temp. Phys. 148, 193 (2007); E. Rousseau, D.Ponarin, L.Hristakos, E.Varoquaux, O.Avenel and Y.Mukharsky, arXiv:0808.0955v1.

3 S.J.Angus, A.J.Ferguson, A.S.Dzurak and R.G.Clark, Appl.Phys.Lett. 90, 112103 (2008).

4 D.Vion, A.Aassime, A.Cottet, P.Joyez, H.Pothier, C.Urbina, D.Esteve, and M.H.Devoret, Science 296, 886 (2002).

${ }^{5}$ D.E.Grupp, T.Zhang, G.J.Dolan and N.S.Wingreen, Phys.Rev.Lett. 87, 186805 (2001).

${ }^{6}$ M.Furlan and S.V.Lotkhov, Phys.Rev. B 67, 205313 (2003).

${ }^{7}$ K.R.Brown, L.Sun and B.E.Kane, Appl.Phys.Lett. 88, 213118 (2006).

${ }^{8}$ G.Ithier, E.Collin, P.Joyez, P.J.Meeson, D.Vion, D.Esteve, F.Chiarello, A.Shnirman, Y.Makhlin, J.Schriefl and G.Schön., Phys.Rev. B 72, 134519 (2005).

${ }^{9}$ C.T.Rogers and R.A.Buhrman, Phys.Rev.Lett. 53, 1272 (1984).

${ }^{10}$ Quantum Tunneling in Condensed Media, (Eds. Yu.Kagan, A.J.Leggett, Elsevier, 1992).

${ }^{11}$ M.I.Dykman, I.B.Schwartz and M.Shapiro, Phys.Rev. E72, 021102 (2005).

${ }^{12}$ S.Shulz, S.Schnüll, C.Heyn and W.Hansen, Phys.Rev. B 69, 195317 (2004).

${ }^{13}$ D.G.Rees, PhD thesis, University of London (2006). 
${ }^{14}$ The SET parameters are: normal state resistance $R_{\mathrm{N}}=30 \mathrm{k} \Omega$; Coulomb energy $E_{\mathrm{C}}=35$ $\mu \mathrm{eV}$; Josephson energy $E_{\mathrm{J}}=20 \mu \mathrm{eV}$; energy gap $2 \Delta=380 \mu \mathrm{eV}$.

${ }^{15}$ P.-M. Billangeon, F.Pierre, H.Bouchiat and R.Deblock, Phys.Rev.Lett. 98, 126802 (2007)

${ }^{16}$ A.Amar, D.Song, C.J.Lobb and F.C.Wellstood, Phys.Rev.Lett. 72, 3234 (1994).

${ }^{17}$ In general, $E \propto v^{\xi}$. We find $\xi=1.0 \pm 0.05$. If the tunneling barrier went to zero, we would expect $\xi=1.5$.

${ }^{18}$ The threshold voltages $V_{-}$and $V_{+}$are determined as fitting parameters to Eq.(2).

${ }^{19}$ The thermal conductance from the SET island varies as $\left(T_{\mathrm{SET}}^{5}-T^{5}\right)$; A.N.Korotkov, M.R.Samuelsen and S.A.Vasenko, J.Appl.Phys. 76, 3623 (1994).

20 T.M.Buehler, V.Chan, A.J.Ferguson, A.S.Dzurak, F.E.Hudson, D.J.Reilly, A.R.Hamilton and R.G.Clark, App.Phys.Lett. 88, 192101 (2006).

${ }^{21}$ T.M.Eiles, J.M.Martinis and M.H.Devoret, Phys.Rev.Lett. 70, 1862 (1993); T.M.Eiles and J.M.Martinis, Phys.Rev. B 50, 627 (1994).

${ }^{22}$ N.M.Zimmerman, B.J.Simonds, A.Fujiwara, Y.Ono, Y.Takahashi, and H.Inokawa, Appl.Phys.Lett. 90, 033507 (2007).

${ }^{23}$ E.Tan, P.G.Mather, A.C.Perrella, J.C.Read and R.A.Buhrman, Phys. Rev. B 71, 161401(R) (2005); P.G.Mather, A.C.Perrella, E.Tan, J.C.Read and R.A.Buhrman, Appl. Phys. Lett. 86, 242504 (2005).

${ }^{24}$ H.R.Zeller and I.Giaever, Phys.Rev. 181, 789 (1969).

${ }^{25}$ D.C.Ralph, C.T.Black and M.Tinkham, Phys.Rev.Lett. 74, 3241 (1995); C.T.Black, D.C.Ralph and M.Tinkham, Phys.Rev.Lett. 76, 688 (1996); D.C.Ralph, C.T.Black and M.Tinkham, Phys.Rev.Lett. 78, 4087 (1997). 
${ }^{26}$ The trap could be a metallic dot, with a Coulomb energy $>>\Delta E$. 
FIG. 1. (Color online) (a) SEM micrograph showing the SET electrodes and island. The source (S), drain (D) and gate (G1, with 4 connected electrodes, and G2) leads are covered by a metallic ground plane. (b) The $I-V$ characteristic, showing the Cooper pair peak (C), the JQP peak (J) and the quasiparticle branch. (c) CBO at 15 mK for up (black, $\square)$ and down (red/grey, •) gate sweeps at the JQP peak $\left(V_{\text {SD }}=\right.$ $0.55 \mathrm{mV}$, lower trace) showing $1 e$ periodicity and at the Cooper pair peak $\left(V_{\mathrm{SD}}=25\right.$ $\mu \mathrm{V}$, upper trace) showing $2 e$ periodicity. The data show a hysteretic transition into a trapped charge state with $\delta Q=0.92 e$.

FIG. 2. Capture and escape rates $1 / \tau$ versus gate voltage $V_{G 2}$ at $25 \mathrm{mK}$. The hysteresis region (A) from -19 to $+37 \mathrm{mV}$ is shown as $1 / \tau=0$. [Inset: Typical exponential decays into and out of a trap for $V_{\mathrm{G} 2}=88 \mathrm{mV}$ (In jump, $\tau=41.2 \mathrm{~ms}$ ) and $V_{\mathrm{G} 2}=-88 \mathrm{mV}($ Out jump, $\left.\tau=65.5 \mathrm{~ms})\right]$.

FIG. 3. (Color online) Electronic transitions when sweeping the gate voltage at $a=$ $0.036 \mathrm{~V} / \mathrm{s}$. The arrow shows the sweep direction. (a) the occupancy of the initial state $P(v, T)$ at $25 \mathrm{mK}(\circ)$ and $165 \mathrm{mK}(\square)$. (b) Logarithmic plots of $P(v, T)$ and $-\log _{10} P(v$, $T$ ) showing the double exponential growth of thermal excitation below the transition $(v<0)$ and the exponential decay above the transition $(v>0)$. Lines show fits to Eq.(2).

FIG. 4. The parameters $\gamma T(\bullet)$ and $\tau_{0}(T)(\Delta)$ versus the refrigerator temperature $T$. The lines show $\gamma T$ (solid) and $\gamma T_{\text {SET }}\left(\right.$ dotted, allowing for heating ${ }^{19}$ ) for $\gamma=145$. 

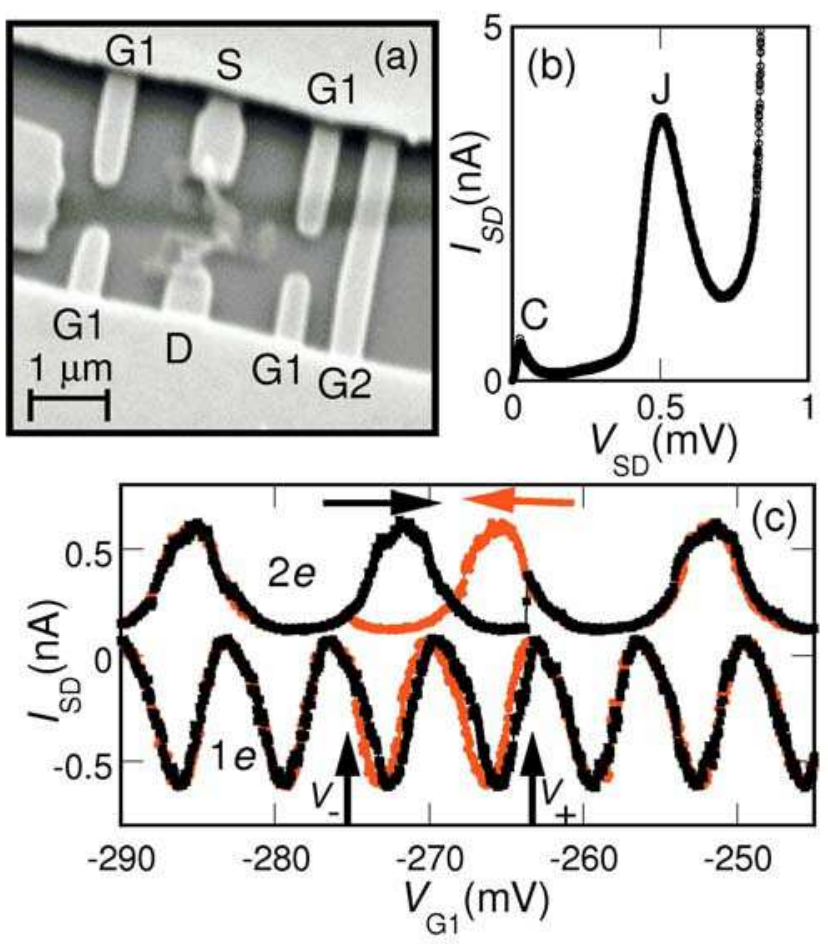

Figure 1 


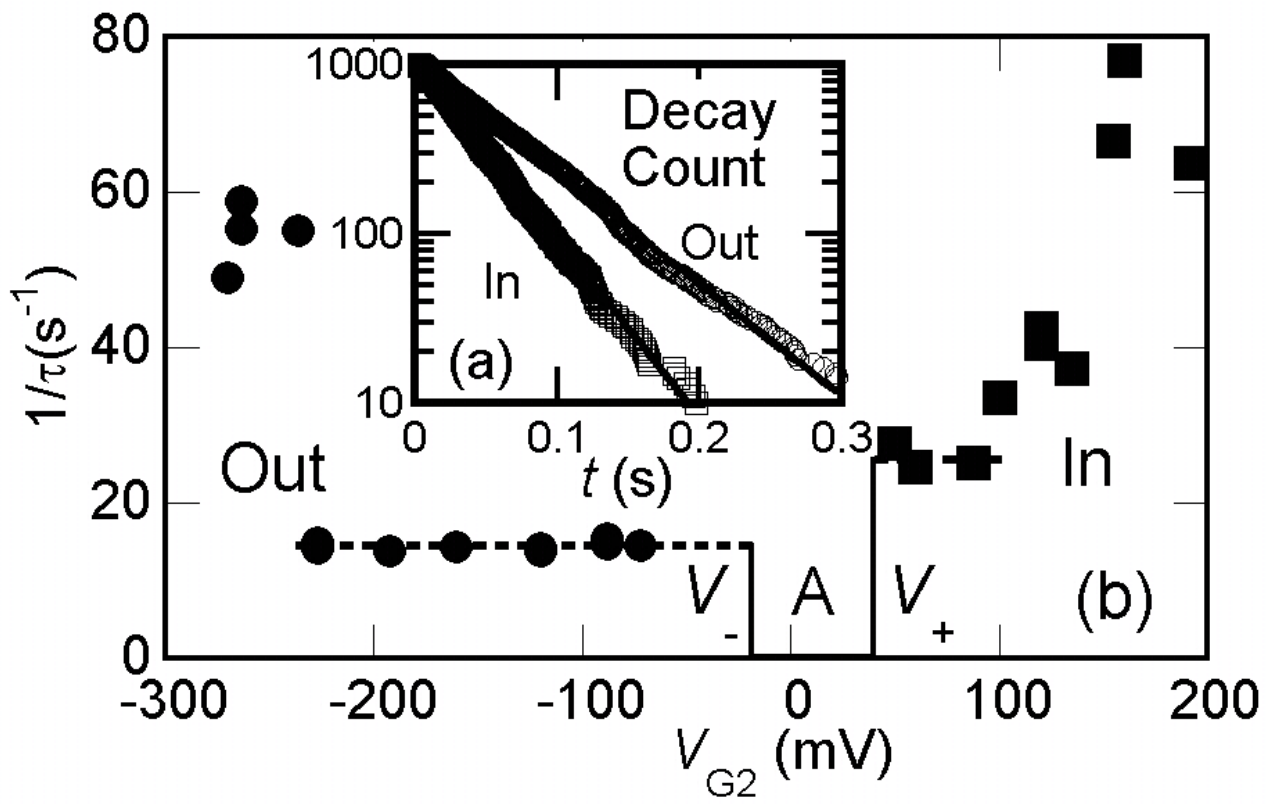

Figure 2 

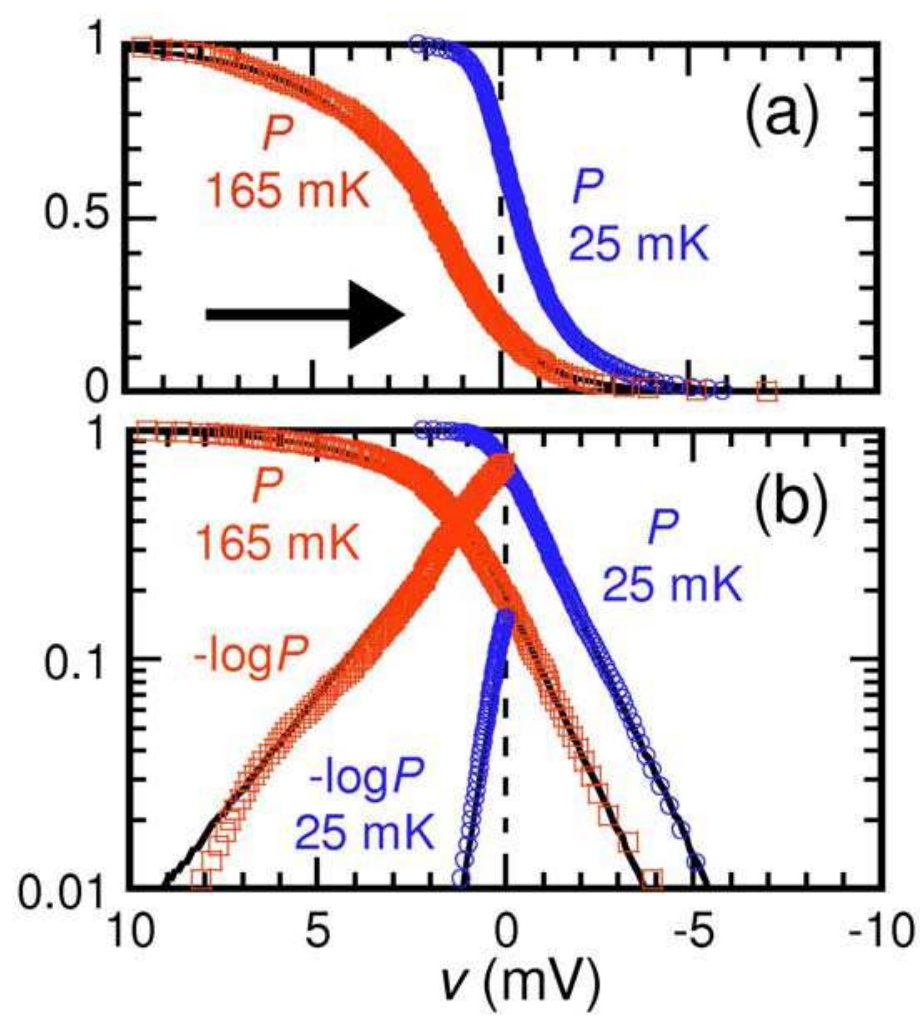

Figure 3 


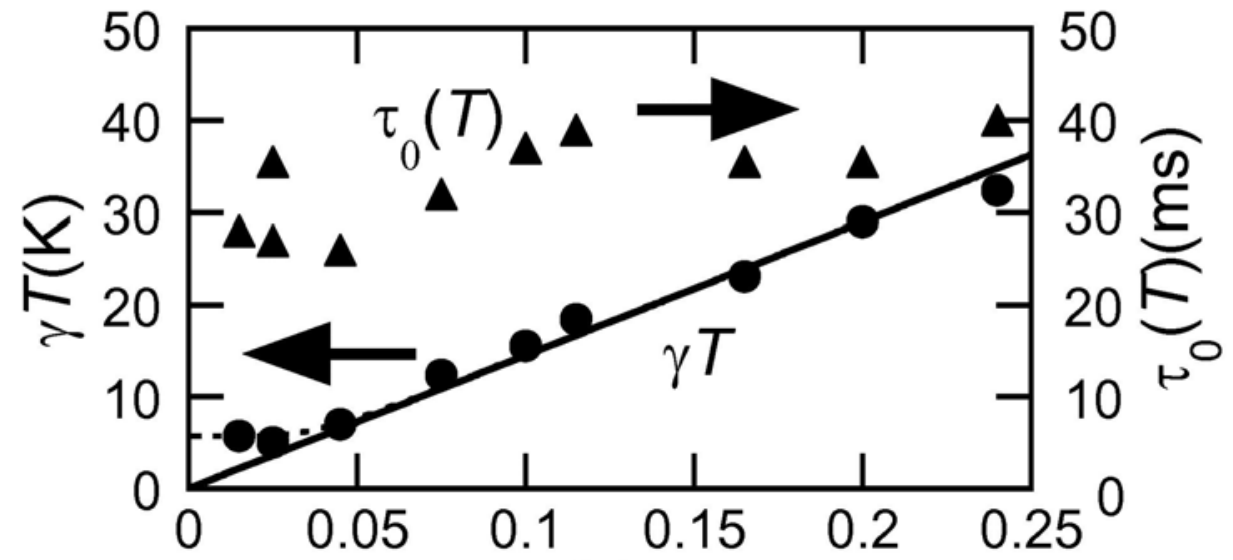

Figure 4 